\title{
Características do uso de benzodiazepínicos por mulheres que buscavam tratamento na atenção primária
}

\author{
Characteristics of the use of benzodiazepines by women seeking treatment in primary care
}

\author{
David Gonçalves Nordon', Karin Akamine², Neil Ferreira Novo³, Carlos von Krakauer Hübner ${ }^{4}$ \\ ${ }^{1}$ Acadêmico, Medicina, Pontifícia Universidade Católica de São Paulo (PUC-SP), São Paulo, SP. ${ }^{2}$ Acadêmica, Medicina, PUC-SP. ${ }^{3}$ Doutor. Professor, Bioestatística, PUC-SP. \\ ${ }^{4}$ Doutor. Professor, Psiquiatria, PUC-SP. \\ Trabalho realizado na Pontifícia Universidade Católica de São Paulo (PUC-SP), São Paulo, SP. \\ Suporte financeiro: Programa Institucional de Bolsas de Iniciação Científica - Conselho de Ensino Pesquisa e Extensão (PIBIC-CEPE).
}

\section{Resumo}

Introdução: Benzodiazepínicos são medicamentos psicotrópicos de prescrição restrita utilizados para crises agudas de ansiedade, insônia e convulsões. Sua utilização é muitas vezes inadequada. O objetivo deste trabalho é identificar o perfil da usuária de benzodiazepínicos na atenção primária e as características de sua utilização.

Método: Foram entrevistadas todas as pacientes maiores de 18 anos em espera para atendimento em uma unidade básica de saúde de Sorocaba (SP) em 2008.

Resultados: Foi encontrada utilização de benzodiazepínicos por $13,14 \%$ das frequentadoras da unidade básica de saúde, sendo maior em mulheres de 50 a 69 anos, em relacionamento estável e analfabetas. O uso encontrado foi principalmente por insônia (48,14\%), de medicamentos de longa meia-vida, crônico (mais de 6 meses, $89,14 \%$ ), com prescrição inicial principalmente pelo clínico geral (47,82\%), com alta taxa de tentativas de interrupção $(91,3 \%)$ infrutíferas $(69,05 \%)$.

Discussão: A utilização é 3,3 vezes maior do que a descrita na literatura para mulheres de perfil semelhante, com prescrições em geral inadequadas, independentemente do prescritor inicial (especialista ou não).

Conclusão: O uso é crônico em mulheres mais idosas, de menor escolaridade, de relacionamento estável, por motivos corretos, porém de tempo de tratamento inadequado.

Descritores: Prescrição de medicamentos, benzodiazepínicos, atenção primária, mulheres.

\begin{abstract}
Introduction: Benzodiazepines are psychotropic drugs whose prescription is indicated for acute crises of anxiety, insomnia and convulsions. Its use is most of the times inadequate. This study aimed at identifying the profile of the female benzodiazepine user at primary care and the characteristics of use.

Methods: All patients over 18 years old were questioned about their benzodiazepine use at a primary care unit from Sorocaba, Brazil, in the year of 2008.

Results: Benzodiazepines were used by $13.14 \%$, mainly by older women (50-69 years old), in a stable relationship and illiterate. The use was mainly due to insomnia (48.14\%), of longer half-life drugs, chronic (over 6 months, $89.14 \%$ ), with first prescription by the general practitioner (47.82\%) and high rates of interruption (91.3\%) failures $(69.05 \%)$.

Discussion: The use is 3.3 times higher than described in the literature for women of similar profiles, with generally inadequate prescriptions, independently of the first prescriber (specialist or not).

Conclusion: Use is chronic, mainly by older women, with lower educational level and who are in a stable relationship, for correct reasons, but for inadequate time of treatment.
\end{abstract}

Keywords: Drugs prescription, benzodiazepines, primary care, women.

Correspondência:

David Gonçalves Nordon, Rua Marechal Castelo Branco, 91/B.03/103, Jardim Sandra, CEP 18031-300, Sorocaba, SP. E-mail: d-nordon@uol.com.br Não foram declarados conflitos de interesse associados à publicação deste artigo.

Copyright (C) Revista de Psiquiatria do Rio Grande do Sul - APRS

Recebido em 27/07/2009. Aceito em 19/11/2009. 


\section{Introdução}

Disponíveis desde 1960 e com um controle rigoroso de sua prescrição devido ao seu potencial de adição, através do formulário azul e da retenção de receita, os benzodiazepíni$\cos (\mathrm{BZD})$ são uma classe dos psicofármacos das mais prescritas atualmente ${ }^{1}$.

No Brasil, é a terceira classe de drogas mais prescritas ${ }^{2}$, sendo utilizada por aproximadamente $4 \%$ da população ${ }^{3-5}$. Hoje em dia, os BZD são indicados apenas para o tratamento agudo e subagudo de ansiedade, insônia e crises convulsivas, embora, no passado, tenham sido usados como primeira linha de tratamento para vários transtornos, principalmente psiquiátricos ${ }^{6}$.

Por geralmente diminuírem o seu efeito ansiolítico ao longo do tempo (em geral 3 a 4 meses), os BZD não são indicados para tratamento de longo prazo e perdem seu lugar para as drogas $\mathrm{Z}$ (agonistas dos receptores do GABA, como zolpidem e zaleplam), que, embora mais caras, têm eficácia semelhante e menos efeitos colaterais ${ }^{7}$. Outro dos fatores preocupantes com relação aos BZD que hoje nos levam a restringir sua prescrição é a capacidade de gerar tolerância e dependência, que podem ser perpetuadas por diversos fatores: prescrição errônea e continuada pelo médico; aumento da dose pelo próprio paciente; necessidade psicológica da droga (algo bastante usual e observado em ambiente ambulatorial). Acredita-se, aliás, que o maior fator atualmente para a perpetuação do hábito seja essa fissura, tendo em vista, como exposto acima, que a dependência química e física de BZD não é tão acentuada quanto de outras possíveis drogas de abuso ${ }^{2,8-13}$.

A tolerância, por outro lado, já é mais difícil de ser encontrada, especialmente em pacientes idosos, os quais a desenvolvem mesmo sem aumentar as doses, por alterações próprias da senescência ${ }^{14}$.

Os usuários de BZD são, em maioria, mulheres (duas a três vezes mais do que homens), e seu número aumenta conforme a idade. No Brasil, é usado principalmente por divorciadas ou viúvas, com menor renda, de 60 a 69 anos de idade $^{2,4,11}$. Seu uso é três vezes mais provável em pacientes portadores de transtornos psiquiátricos ${ }^{15}$.

A prescrição desses fármacos, em geral, também é inadequada, em especial no nível primário de atendimento, de acordo com diversos artigos ${ }^{16-23}$. Os principais motivos para tal são a falta de tempo, a subestimação da quantidade de usuários, da gravidade do uso, dos efeitos colaterais e até mesmo a não observação dos guidelines.

O Brasil carece de dados a respeito da utilização de BZD, em especial para a população que se utiliza de unidades básicas de saúde (UBS), o pilar do atendimento primário. Deste modo, é interessante para a saúde pública a análise dos usuários, seu perfil socioeconômico e de uso, além da adequabilidade da prescrição, neste cenário frequentado principalmente por mulheres.

\section{Método}

Foram entrevistadas, ao longo do ano de 2008 (janeiro a dezembro), às sextas-feiras de manhã, pelos dois pesquisadores, acadêmicos de medicina da Pontifícia Universidade Católica de São Paulo, as pacientes que se encontravam na fila de espera para o atendimento na UBS da Vila Barão, em Sorocaba, SP, maiores de 18 anos e que concordaram em participar e assinar o termo de consentimento livre e esclarecido (TCLE). Os critérios de inclusão foram: concordar em participar e assinar o TCLE; ser mulher; ser maior de idade; estar devidamente inscrita na UBS, mediante ficha e número de registro.

A UBS da Vila Barão é uma pequena UBS em um bairro da periferia de Sorocaba, que atende ao redor de 14.000 pessoas, incluindo neste número uma grande porção de moradores de terrenos não loteados (área verde). É composta por uma população em média de baixa renda e escolaridade. A UBS conta com os serviços de clínica geral, pediatria e ginecologia apenas, não contando com outras facilidades como ortodontia, psicologia ou psiquiatria.

As entrevistadas responderam a uma ficha de perguntas elaborada pelos pesquisadores para este devido fim, preenchida pelos pesquisadores com nome, idade, estado civil, escolaridade, renda per capita em salários-mínimos, e se utilizavam, ou não, BZD. As usuárias, então, respondiam a uma segunda bateria de perguntas, a respeito da característica do uso: 1) benzodiazepínico utilizado; 2) motivo de uso (autorreferido); 3 ) tempo de uso; 4) primeiro prescritor da receita; 5) prescritor atual; 6) tentativas prévias de interrupção de uso; 7) sucesso ou insucesso nessa interrupção; e 8) motivo do insucesso na interrupção.

As entrevistadas foram, então, agrupadas pelas características: 1) idade: de 18 a 29 anos; de 30 a 39 anos; de 40 a 49 anos; de 50 a 59 anos; de 60 a 69 anos; e 70 anos ou mais; 2) estado civil: casada, solteira, viúva ou amigada; 3 ) escolaridade: analfabeta, até a $8^{a}$ série do ensino fundamental, e ensino médio/superior; e 4) renda familiar per capita: até três salários-mínimos e quatro ou mais salários-mínimos.

Para análise dos resultados, aplicou-se o teste do quiquadrado ou teste exato de Fisher ${ }^{24}$, com o objetivo de comparar grupos etários, estado civil, escolaridade e outras características em relação ao uso, ou não, dos BZD. Em todos os testes fixou-se em 0,05 ou $5 \%$ o nível de significância.

Este trabalho foi aprovado pelo comitê de ética em pesquisa do Centro de Ciências Médicas e Biológicas da Pontifícia Universidade Católica de São Paulo (PUC-SP), São Paulo (SP), em 12/06/07.

\section{Resultados}

Ao longo do ano de 2008, foram entrevistadas na UBS da Vila Barão, em Sorocaba, 350 mulheres (3\% da população total, incluindo homens e mulheres, atingida pela UBS, aproximadamente). Destas, $46(13,14 \%)$ eram usuárias de 
BZD, e 304 (86,86\%) não eram, destoando significativamente dos dados internacionais, que mostram uma utilização por aproximadamente $4 \%$ da população geral ${ }^{3-5}$.
As respostas dos itens do questionário de pesquisa estão quantificadas na Tabela 1 para as frequentadoras em geral, e de 2 a 3 para as usuárias, exclusivamente.

Tabela 1 - Características demográficas das entrevistadas (idade, estado civil, escolaridade e renda per capita) de acordo com a utilização de benzodiazepínicos

\begin{tabular}{lcc}
\hline População/variável & Porcentagem de usuárias & Qui-quadrado \\
\hline Idade & 2,7 & $31,89^{*}$ \\
$18-29$ & 7,8 & \\
$30-39$ & 19,0 & \\
$40-49$ & 29,7 & $7,33 \dagger$ \\
$50-59$ & 29,4 & \\
$60-69$ & 18,8 & $11,68+$ \\
70 e maiores & & \\
Estado civil & 17,7 & \\
Casada & 9,8 & \\
Solteira & 9,4 & \\
Viúva & 5,4 & - \\
Amigada & & \\
Escolaridade & 28,6 & \\
Analfabeta & 14,4 & \\
Até a $8^{a}$ série & 8 & \\
Ensino médio/superior & & \\
Renda per capita (salários-mínimos) & 14,2 & \\
Até 3 & 3,8 & \\
4 ou mais & 13,14 & \\
Total de usuárias &
\end{tabular}

$* \mathrm{p}<0,001(18-39<40$ ou mais $)$.

$\dagger$ Não significante.

$\ddagger \mathrm{p}<0,05$ (analfabetas $>$ outras).

Como observado na Tabela 1, o grupo etário de mulheres de 18 a 39 anos (200 pessoas, ou $57 \%$ da amostra) apresenta um consumo de BZD significantemente menor do que o grupo de 50 a 69 anos $(\mathrm{p}<0,001)$.

Os resultados demonstrando o estado civil das frequentadoras apenas sugerem que mulheres em um relacionamento estável (casadas ou amigadas; 67,42\%) consomem mais do que mulheres solteiras ou viúvas, uma vez que a análise estatística não foi significativa.

A escolaridade das frequentadoras e das usuárias é mostrada em seguida; mulheres analfabetas consomem significantemente mais BZD do que mulheres com escolaridade mais alta $(\mathrm{p}<0,05)$.

A Tabela 1 ainda mostra a renda familiar per capita estimada das frequentadoras, autorreferida; os dados, porém, não foram estatisticamente significativos, com apenas uma leve sugestão de que mulheres com menor renda utilizam mais BZD.

A Tabela 2 mostra todos os resultados do nosso levantamento, com o número de respostas e a porcentagem da população usuária correspondente.
O maior motivo de utilização (que poderia ser mais de um) foi insônia (48,14\%), seguido por ansiedade $(39,5 \%)$ e convulsões/epilepsia $(8,64 \%)$. Duas mulheres deram como motivo o tratamento de depressão, e uma, luto (mas não ansiedade).

Quanto aos medicamentos em uso, o mais citado foi o diazepam $(76,08 \%)$, seguido pelo clonazepam $(8,68 \%)$ e a terapia combinada entre os dois $(6,52 \%)$, ambos adquiriveis na UBS. Quatro mulheres relataram o uso de outros BZD que não estes, não adquiríveis pelo sistema público de saúde de Sorocaba.

O tempo de uso foi prolongado (maior que 6 meses) em $89,14 \%$ da população estudada.

O principal prescritor inicial é o clínico geral $(47,82 \%$ das usuárias), seguido pelo psiquiatra $(36,95 \%)$. Nenhuma usuária obteve sua primeira prescrição através de um farmacêutico; contudo, uma usuária ainda relatou ter recebido sua prescrição inicial pelo cardiologista, outra pelo endocrinologista e três pelo neurologista $(6,52 \%)$.

Para a maioria $(65,21 \%)$, o principal prescritor atual é o clínico geral do posto que frequentam, que muitas vezes simplesmente mantém uma receita anterior. A prescrição por psiquiatras, porém, se reduz pouco e não significativamente 
Tabela 2 - Motivo de uso do benzodiazepínico, medicamento utilizado, primeiro prescritor, prescritor atual, tentativas prévias de interrupção do uso, sucesso na tentativa, razão do insucesso - total de respostas e porcentagem da população de usuárias

\begin{tabular}{|c|c|}
\hline Variável & Porcentagem \\
\hline \multicolumn{2}{|l|}{ Motivo do uso } \\
\hline Ansiedade & 39,5 \\
\hline Insônia & 48,1 \\
\hline Convulsões/epilepsia & 8,6 \\
\hline “Depressão" & 2,5 \\
\hline Luto & 1,2 \\
\hline \multicolumn{2}{|l|}{ Medicamento em uso } \\
\hline Diazepam & 76,1 \\
\hline Clonazepam & 8,7 \\
\hline Diazepam + clonazepam & 6,5 \\
\hline Lorazepam & 2,2 \\
\hline Bromazebam & 2,2 \\
\hline Nitrazepam & 4,3 \\
\hline \multicolumn{2}{|l|}{ Tempo de utilização } \\
\hline Até 5 meses & 10,9 \\
\hline De 6 a 11 meses & 4,3 \\
\hline De 12 a 35 meses & 15,2 \\
\hline Mais de 36 meses & 69,6 \\
\hline \multicolumn{2}{|l|}{ Primeiro prescritor } \\
\hline Clínico geral & 47,8 \\
\hline Psiquiatra & 37 \\
\hline Farmacêutico & 0 \\
\hline Amigos/parentes & 2,2 \\
\hline Outros médicos & 10,9 \\
\hline Não lembra & 2,2 \\
\hline \multicolumn{2}{|l|}{ Prescritor atual } \\
\hline Clínico geral & 65,2 \\
\hline Psiquiatria & 30,4 \\
\hline Cardiologista & 2,2 \\
\hline Pega comprimidos de parente & 2,2 \\
\hline \multicolumn{2}{|l|}{ Tentativas prévias de interrupção do uso } \\
\hline Sim & 91,3 \\
\hline Não & 8,7 \\
\hline \multicolumn{2}{|l|}{ Sucesso na tentativa } \\
\hline Sim & 30,95 \\
\hline Não & 69,05 \\
\hline \multicolumn{2}{|l|}{ Motivo do insucesso } \\
\hline Persistência dos sintomas anteriores ao uso & 90,6 \\
\hline Ansiedade e paresia/parestesias noturnas & 3,1 \\
\hline Morte de um parente & 3,1 \\
\hline Médico não deixou & 3,1 \\
\hline Total & 100,0 \\
\hline
\end{tabular}

(aproximadamente 10\%) com relação à prescrição inicial, enquanto a de outros especialistas se resume a apenas um cardiologista, em detrimento do clínico geral inicial.

A ampla maioria das mulheres (91,3\%) tentou interromper a utilização. Foi obtido sucesso em apenas 30,95\% da amostra; o principal motivo, na grande maioria, foi a persistência dos sintomas relatados antes do uso. Outras queixas foram relata- das, dentre elas a morte de um parente (uma usuária) e a não permissão, por parte do médico, da interrupção do tratamento (uma usuária). Tal usuária alegou a utilização da droga para ansiedade, insônia e convulsões, e fazia uso já havia mais de 3 anos, por prescrição inicial e atual de um psiquiatra.

A Tabela 3 mostra as respostas das usuárias de acordo com o seu prescritor inicial. 
Tabela 3 - Motivo do uso do benzodiazepínico e tempo de uso de acordo com o prescritor inicial

\begin{tabular}{lcc}
\hline & \multicolumn{2}{c}{ Prescritor inicial } \\
\cline { 2 - 3 } Variável & Psiquiatra, n (\%) & Clínico geral, n (\%) \\
\hline Motivo do uso & $0(0)$ & $3(14,3)$ \\
Ansiedade & $1(6,2)$ & $9(42,9)$ \\
Insônia & $15(93,8)$ & $9(42,9)$ \\
Ambos & $16(100)$ & $21(100)$ \\
Total & $1(5,9)$ & $4(18,2)$ \\
Tempo de uso & $16(94,1)$ & $18(81,8)$ \\
$<6$ meses & $17(100)$ & $22(100)$ \\
6 meses & & 0,2617 \\
Total &
\end{tabular}

* Qui-quadrado $=7,96$.

O principal motivo de prescrição isolada pelos clínicos gerais foi insônia (40,9\% das usuárias); quando associada, vinha geralmente com ansiedade (40,9\% das usuárias). O clínico geral prescreveu BZD para apenas dois casos de convulsões, um acompanhado de insônia, e outro de ansiedade. Por outro lado, para os psiquiatras, a insônia geralmente não era um fator isolado (apenas 5,88\% das usuárias), mas sim acompanhante de outras queixas, como ansiedade e convulsão (82,35\% das usuárias). É notável que, das que relataram convulsão como a causa inicial da prescrição, seis foram tratadas pelo psiquiatra, duas pelo clínico geral e nenhuma pelo neurologista, o especialista de indicação para tal.

Depressão foi outro motivo citado, sendo tratada inicialmente pelo psiquiatra em dois casos, que ainda hoje recebem prescrição deste, em um tratamento de mais de 3 anos e, no outro caso, por um clínico geral (que não é o especialista indicado para tal).

\section{Discussão}

Os dados obtidos através deste estudo refletem as características daquele bairro e também do próprio atendimento primário em si, enquanto os estudos que foram usados para comparação apresentam dados obtidos através de médias e estudos multicêntricos, o que tanto homogeneíza a amostra como a afasta da realidade (os autores não encontraram equivalentes no Brasil para a comparação de dados). Diante disso, explica-se a diferença de resultados com relação ao uso, muito mais elevado na UBS (3,3 vezes mais do que o encontrado na literatura internacional). Com relação à literatura nacional, um estudo de 1988 da população do Rio de Janeiro $(\mathrm{RJ})^{4}$ demonstrou um uso nos últimos 30 dias de psicotrópicos por $6,7 \%$ das mulheres, sendo $85,23 \%$ destes BZD, o que representaria, ainda, menos da metade do valor encontrado neste estudo. Há, contudo, um estudo semelhante a este, de atenção primária da Espanha ${ }^{16}$, que em 1997 encontrou um risco relativo de 1,57 para o uso de BZD por mulheres, em comparação com homens, totalizando $11 \%$, número semelhante ao deste estudo.

Além disso, aventou-se a hipótese de que o consumo seja aumentado nesta população de estudo devido a vários fatores característicos do local estudado: ambiente e interações sociais (sendo a Vila Barão uma região da periferia, com índices mais altos de violência, quando comparados a outros bairros de Sorocaba) e maior desinformação dos médicos que trabalham em postos de saúde, talvez por menor estímulo à atualização, entre outros fatores, além de outros fatores já citados que alteram a prescrição a nível primário ${ }^{16-23}$.

A faixa etária de maior utilização condiz com o descrito na literatura, embora a tendência observada seja de ser ainda maior em mulheres mais idosas, de 60 a 69 anos de idade $4,16,20-22$, enquanto a deste estudo demonstrou uma faixa etária mais ampla (de 50 a 69 anos). Foi encontrado que mulheres em relacionamento estável apresentam maior tendência de uso, o que concorda com o estudo espanhol de atenção primária $^{16}$ e com um estudo italiano de um serviço específico de saúde mental ${ }^{25}$, mas discorda dos dados do estudo cario$\mathrm{ca}^{4}$, que apresenta maior uso por viúvas.

Foi encontrada uma associação estatisticamente significativa entre analfabetismo e maior uso de BZD, mas não entre menor renda e maior uso, o que pode ser explicado pela baixa renda em geral da população estudada, não sendo significativa a amostra de maior renda. $\mathrm{O}$ estudo do Rio de Janeiro ${ }^{4}$ também encontrou uma associação entre menor renda e escolaridade e maior uso; o estudo espanhol ${ }^{16}$, igualmente, concorda com estes dois com relação ao tempo de estudo, mas o italiano ${ }^{25}$ encontrou uma diferença menos significativa, uma vez que separou a escolaridade em maior ou menor que 8 anos de estudo, ao contrário dos outros estudos, que separaram em maiores categorias, como este.

Isso demonstra uma correlação perigosa, em que pessoas menos informadas e com menor poder aquisitivo acabam recorrendo a um uso de medicamentos para, muitas vezes, resolver problemas psicossociais que poderiam ser resolvidos de outra forma. 
Os principais motivos de utilização relatados pelas pacientes foram queixas para as quais o uso prolongado de BZD não é indicado, mas sim somente para o uso agudo ${ }^{6}$. Como demonstrado anteriormente, para praticamente todas as usuárias $(89,14 \%)$ o uso era crônico e, portanto, inadequado, muito provavelmente devido à desinformação, ou pelo fato de os médicos da UBS, ao criarem um relacionamento afetuoso com seus pacientes, terem certo receio de lhes negar os medicamentos, comumente tão requisitados, como descrito na literatura ${ }^{15,17,20}$. Investigação tão aprofundada, contudo, não foi o escopo desta pesquisa.

É importante ressaltar que, embora possam ser usados controladamente BZD para diminuição dos efeitos colaterais dos remédios antidepressivos no início do tratamento para síndrome do pânico, eles não são de forma alguma indicados diretamente para o transtorno depressivo; com relação ao luto, por ser uma alteração teoricamente autolimitada, os BZD poderiam ser usados (embora não haja indicação expressa para tal) para o controle agudo dos sintomas ansiosos ou insônia, mas deveriam ser retirados tão logo estes cedessem, o que não é observado na usuária crônica que citou o luto como motivo inicial de prescrição pelo clínico geral.

Com relação ao medicamento de escolha, foram receitados praticamente apenas os dispensáveis nas UBS de Sorocaba, que são, infelizmente, BZD de meia-vida longa e, portanto, mais propensos a efeitos colaterais, de modo que esse erro de prescrição não pode se dever ao médico, mas sim ao sistema de saúde. É importante ressaltar que o uso de BZD de meia-vida longa, em especial para idosos, pode ser perigoso, devido aos efeitos colaterais dos seus metabólitos, que demoram mais a deixar o corpo.

A alta incidência de tentativas de interrupção do uso dos BZD pode ser um reflexo do próprio motivo de uso: a queixa de ansiedade é a segunda mais prevalente (39,5\%), algo relativamente controlável de acordo com as modificações psicossociais. A insônia, também, pode ser uma queixa intermitente (e controlável de acordo com a ansiedade), o que levaria a uma maior interrupção do uso crônico. Além disso, é interessante notar que $100 \%$ das pacientes convulsivas tentaram interromper o tratamento, e apenas uma conseguiu - parcialmente; usuária de terapia combinada, ela parou de usar um dos medicamentos (clonazepam) apenas -, mostrando que a indicação do medicamento não só é inadequada como mal orientada. Do mesmo modo, a taxa de sucesso na interrupção foi baixa, o que pode se dever a uma provável dependência dos usuários por tal medicamento, muitas vezes já psicológica, como descrito na literatura.

Não obstante, as tentativas de interrupção são um bom sinal; indicam que a população tem consciência das consequências do uso contínuo de BZD, algo que deveria ser aproveitado pelos médicos responsáveis para a adequação do tratamento.

O ideal seria que o prescritor inicial fosse o psiquiatra, especialista neste tipo de medicação; contudo, sendo este um cenário de atenção primária, é natural que quase metade das prescrições $(47,82 \%)$ tenha sido feita por clínico geral. O estudo do Rio de Janeiro ${ }^{4}$ encontrou uma prescrição por não especialistas (neurologistas/psiquiatras) de 68,5\%. Do mesmo modo, a continuação do tratamento na atenção primária ou a transferência de um serviço secundário/terciário para um primário é natural.

Seguindo essa avaliação, o uso crônico, aqui estabelecido como por mais de 6 meses, foi observado com alta prevalência para todos os prescritores $(94,11 \%$ para psiquiatras e $81,81 \%$ para clínicos gerais), sendo aparentemente mais prevalente entre os psiquiatras, mas não estatisticamente significativo $(\mathrm{p}=$ 0,2617 ). Infelizmente, para a usuária que obtém medicamento sem receita, o seu uso também é crônico. Como já mencionado, os BZD não deveriam ser usados por mais de 3 a 4 meses, pela $p$ erda de sua função ansiolítica e contra a insônia e pelos possíveis efeitos colaterais que seu uso pode trazer a longo prazo (perda cognitiva, diminuição da produtividade, maior possibilidade de acidentes de trânsito).

Quase a metade $(41,17 \%)$ dos pacientes atendidos inicialmente pelo psiquiatra rumou para o clínico geral, refletindo contra-referência de um serviço de especialidade para um serviço primário. Uma usuária foi do psiquiatra para o cardiologista; dentre os que receberam a primeira prescrição do clínico geral, a tendência foi se manter com ele, excetuando-se três usuárias $(13,63 \%)$, que foram referenciadas para um acompanhamento especializado. Observa-se, assim, um atendimento que envolve muitas vezes a simples manutenção da receita e a indicação por outro profissional, sem um acompanhamento especializado. E, mesmo dentre os que se mantiveram com o atendimento especializado, não houve diferença com relação ao tempo de uso, ou seja: a utilização de medicamentos com meia-vida longa é crônica e, portanto, sujeita a maior tolerância, dependência e efeitos colaterais, independentemente da especialidade do prescritor.

Contudo, este estudo possui algumas limitações: limitase à realidade feminina de um atendimento primário em um bairro da periferia, não podendo seus resultados ser expandidos para outros cenários; o motivo de uso foi autorreferido e, portanto, passível de alterações de acordo com o entendimento da usuária, de modo que a adequabilidade da indicação não pôde ser avaliada com total confiança (o que não foi o objetivo inicial da pesquisa) - outros dados a respeito da prescrição, no entanto, são avaliáveis com boa confiança; por fim, não há estudos semelhantes em nosso cenário de atenção primária para podermos comparar os dados, sendo necessárias comparações com escassas fontes internacionais.

\section{Conclusão}

Com este trabalho, foi observado o padrão de uso de BZD por mulheres em uma UBS, refletindo as características de uma população de baixa renda e escolaridade, na qual um número mais de três vezes maior do que a literatura mundial relata é de consumidoras, sendo as principais da faixa etária 
de 50 a 69 anos, em relacionamento estável e analfabetas. A utilização de BZD de meia-vida longa é crônica e, portanto, com maiores efeitos colaterais, por razões errôneas, com prescrições por médicos não indicados primeiramente para tal função, com alto índice de insucesso na tentativa de interrupção do uso. Os prescritores em geral são clínicos gerais, mas mesmo os especialistas se mostraram prescritores inadequados, utilizando BZD de meia-vida longa, por tempo prolongado, sem seguir as normas de conduta ${ }^{6}$ e para queixas que muitas vezes poderiam ser controladas de outras maneiras, como uma maior ação psicossocial.

Os autores acreditam que este trabalho contribui um pouco mais para os conhecimentos das características da população feminina atendida pelo serviço primário de saúde em bairros carentes, não exclusivamente de Sorocaba, mas de toda a região. A necessidade de mais estudos a respeito, tendo em vista as danosas consequências do uso prolongado de BZD, são imperiosas, assim como a orientação e reciclagem periódica dos profissionais de saúde de todas as especialidades a respeito do uso, abuso, dependência e efeitos colaterais dos BZD.

\section{Referências}

1. Rosenbaum JF. Attitudes toward benzodiazepines over the years. J Clin Psychiatry. 2005;66 Suppl 2:4-8.

2. Kapczinski F, Amaral OB, Madruga M, Quevedo J, Busnello JV, de Lima MS. Use and misuse of benzodiazepine in Brazil: a review. Subst Use Misuse. 2001;36(8):1053-69.

3. Galduróz JC, Noto AR, Nappo SA, Carlini EA. Uso de drogas psicotrópicas no Brasil: pesquisa domiciliar envolvendo as 107 maiores cidades do país - 2001 . Rev Latinoam Enferm. 2005;13(n.esp):888-95.

4. Almeida LM, Coutinho ES, Pepe VL. Consumo de psicofármacos em uma região administrativa do Rio de Janeiro: a Ilha do Governador. Cad Saude Publica. 1994;10(1):5-16.

5. Centro Brasileiro de Informações sobre Drogas Psicotrópicas (CEBRID). II levantamento domiciliar sobre o uso de drogas psicotrópicas no Brasil: estudo envolvendo as 108 maiores cidades do país. 2005.

6. Salzman C (Task Force Chair). Benzodiazepine dependence, toxicity, and abuse: a task force report of the American Psychiatric Association. Washington: American Psychiatric Press; 1990.

7. Silva AB. Medicina do sono. In: do Prado FC, Ramos J, do Valle JR. Atualização Terapêutica. $23^{\mathrm{a}}$ edição. São Paulo: Artes Médicas; 2007.
8. Kan CC, Hilberink SR, Breteler MH. Determination of the main risk factors for benzodiazepine dependence using a multivariate and multidimensional approach. Compr Psychiatry. 2004;45(2):88-94

9. Khong E, Sim MG, Hulse G. Benzodiazepine dependence. Aust Fam Physician. 2004;33(11):923-6.

10. O'Brien CP. Benzodiazepine use, abuse and dependence. J Clin Psychiatry. 2005;66 Suppl 2:28-33.

11. Karniol IG, Botega NJ, Maciel RR, Moreira ME, de Capitani EM, de Madureira PR, et al. Uso e abuso de benzodiazepinas no Brasil. Rev ABP-APAL. 1986;8(1):30-5.

12. Rada P, Hoebel BG. Acetylcholine in the accumbens is decreased by diazepam and increased by benzodiazepine withdrawal: a possible mechanism for dependency. Eur J Pharmacol. 2005;508(1-3):131-8.

13. Allison C, Pratt JA. Neuroadaptive processes in GABAergic and glutamatergic systems in benzodiazepine dependence. Pharmacol Ther. 2003;98(2):171-95

14. Voyer P, McCubbin M, Cohen D, Lauzon S, Collin J, Boivin C. Unconventional indicators of drug dependence among elderly long-term users of benzodiazepines. Issues Ment Health Nurs. 2004;25(6):603-28.

15. González Rubio MI, Rojas Castillo G, Díaz Vargas B. Uso de psicofármacos por consultantes al nivel primario. Rev Psiquiatr (Santiago de Chile). 1995;12(3/4):186-9.

16. Escrivá R, Pérez A, Lumbreras C, Molina J, Sanz T, Corral MA. Prescripción de benzodiacepinas en un centro de salud: prevalencia, cómo es su consumo y características del consumidor. Aten Primaria. 2000;25(2):107-10.

17. Straand J, Rokstad K. General practitioners' prescribing patterns of benzodiazepine hypnotics: are elderly patients at particular risk for overprescribing? A report from the More \& Romsdal Prescription Study. Scand J Prim Health Care. 1997;15(1):16-21.

18. Bendtsen P, Hensing G, McKenzie L, Stridsman AK. Prescribing benzodiazepines--a critical incident study of a physician dilemma. Soc Sci Med. 1999;49(4):459-67.

19. Monette J, Tamblyn RM, McLeod PJ, Gayton DC. Characteristics of physicians who frequently prescribe long-acting benzodiazepines for the elderly. Eval Health Prof. 1997;20(2):115-30.

20. Álvarez TF, Castro MJG, Morente CB, Fernández JM. Factores que influyen en la prescripción de benzodiacepinas y acciones para mejorar su uso: un estudio Delphi en médicos de atención primaria. Aten Primaria. 2002;30(5):297-303.

21. Anthierens S, Habraken H, Petrovic M., Christiaens T. The lesser evil? Initiating a benzodiazepine prescription in general practice: a qualitative study on GPs perspectives. Scand J Prim Health Care. 2007;25(4):214-9.

22. Cook JM, Marshall R, Masci C, Coyne JC. Physicians' perspectives on prescribing benzodiazepines for older adults: a qualitative study. J Gen Intern Med. 2007;22(3):303-7.

23. van Risjwijk E, Borghius M, van de Lisdnok E, Zitman F, van Weel C. Treatment of mental health problems in general practice: a survey of psychotropics prescribed and other treatments provided. Int J Clin Pharmacol Ther. 2007;45(1):23-9.

24. Siegel SE, Castellan JR, NJ. Estatística não paramétrica para ciências do comprotamento. 2a ed. Porto Alegre: Artmed; 2006. p. 448.

25. Veronese A, Garatti M, Cipriani A, Barbui C. Benzodiazepine use in the real world of psychiatric practice : low-dose,long-term drug taking and low rates of treatment discontinuation. Eur J Clin Pharmacol, 2007;63(9):867-73. 